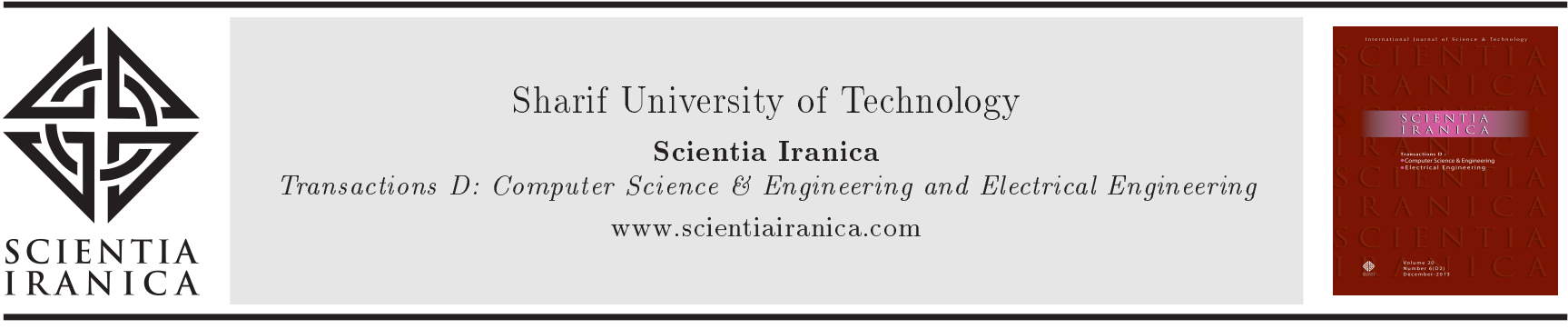

\title{
The Portable Neuromodulation Stimulator (PoNS) for neurorehabilitation
}

\author{
K.A. Kaczmarek* \\ University of Wisconsin-Madison, 1550 Engineering Drive, Room 2156, Madison, Wisconsin 53706, USA. \\ Received 29 August 2015; received in revised form 4 May 2016; accepted 5 September 2016
}

\author{
KEYWORDS \\ Electrocutaneous; \\ Electrotactile; \\ Neuromodulation; \\ Neuroplasticity; \\ Neurorehabilitation; \\ Tongue.
}

\begin{abstract}
The Portable Neuromodulation Stimulator (PoNS) is a compact, self-contained device that delivers a fixed sequence of dc-balanced voltage pulses to the anterior-dorsal tongue through a matrix of 143 gold-plated electrodes. This form of stimulation is being investigated as a possible aid to rehabilitation of motor, cognitive, and emotional symptoms resulting from a range of neurological disorders of traumatic, degenerative, or developmental origin. This article provides a technical overview of the PoNS device as well as a summary of applications research to date.

(C) 2017 Sharif University of Technology. All rights reserved.
\end{abstract}

\section{Introduction}

This paper describes the theory, design, and application of the Portable Neuromodulation Stimulator (PoNS ${ }^{\top \mathrm{M}}$; Figure 1), a device developed by the author in 2008 to facilitate collaborative studies examining the effects of comfortable electrical stimulation of the tongue on the efficacy of neurorehabilitation exercises. Some design elements of the PoNS device are based on the earlier Tongue Display Unit (TDU; Figure 1), which is a general-purpose, programmable platform for investigation of tongue electrotactile psychophysics as well as for development of potential applications of tactile information display on the tongue [1-3]. A full technical description of the TDU, as well as a summary of research using it, appears in [4].

The conceptual framework leading to PoNS development resulted from adventitious observations using the TDU, which, unlike the PoNS device, could modulate the tongue stimulation in real time in response to external inputs. For example, when head tilt

\footnotetext{
*. Tel.: 608 265-3756

E-mail address: kurt.kaczmarek@wisc.edu
}

about the anterior-posterior and lateral-medial axes was mapped to a spatially-corresponding electrotactile stimulus on the ventral tongue surface of vestibularimpaired individuals, not only immediate postural stability improvement was observed, but also a longerterm (minutes to months) balance improvement, even after the apparatus was removed from the mouth [5,6]. Subsequent technology license resulted in the BrainPort $^{\mathrm{TM}}$ balance and vision sensory substitution devices by Wicab, Inc. (http://www.wicab.com). The TDU itself was never commercialized.

The sustained benefits of tongue-based sensory substitution lead to the question of whether the supplemental balance information is necessary, or whether the electrotactile stimulus alone on the tongue may be beneficial. Indeed, independent experiments at the University of Wisconsin-Madison have revealed that balance exercises combined with whole-array tongue stimulation (with no modulation based on head position or any other exogenous variable) lead to both longterm balance improvement (greater than that typically observed by exercises alone) as well as normalization of activity in brain circuits responsible for postural control [7-15]. Collectively these results, using both the TDU (programmed to deliver steady, unmodulated tongue stimulation) and the similar stimulation us- 


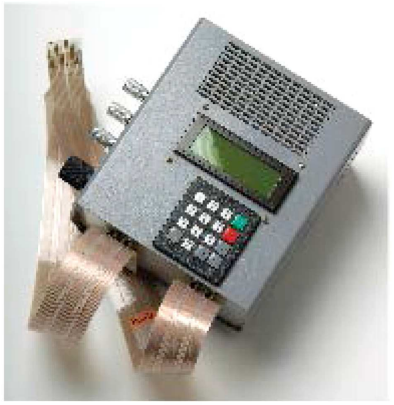

(a)

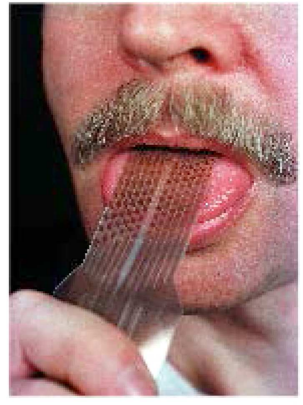

(b)

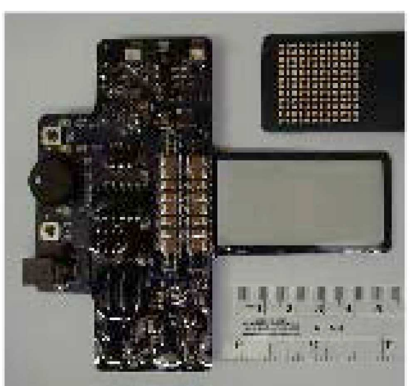

(c)

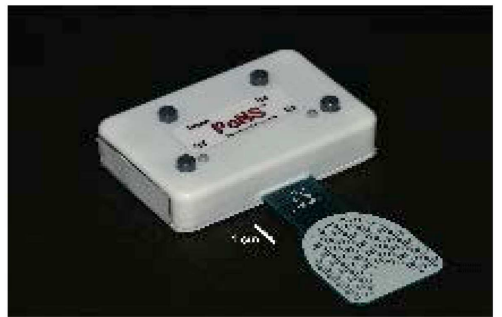

(d)

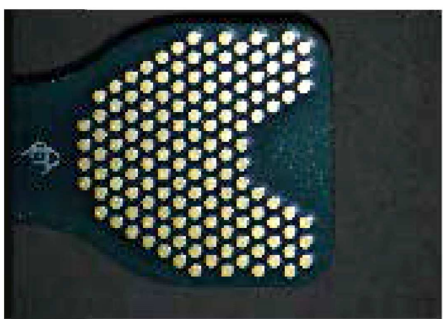

(e)

Figure 1. Tongue stimulation instrumentation: (a) Tongue Display Unit (TDU, developed in 1999), (b) electrode array for TDU, (c) PoNS version 1 (2009), (d) PoNS version 2 (2011), and (e) electrode array for PoNS version 2 (on bottom side of PoNS circuit board)

ing the PoNS device, suggest that information-devoid tongue stimulation appears to improve the effects of the exercises via beneficial neuroplasticity. Selected results from these studies appear later in this paper.

Given the size, fragility, and operational complexity of the TDU, we developed the PoNS family of devices as a platform to examine the mechanisms of recovery and optimization of methods combining tongue stimulation and therapeutic exercises. This has also enabled expanding interventions into the homes of subjects by providing each with a PoNS device during the study. Because the TDU and PoNS share several similarities, emphasis will be given to features and characteristics specific to the latter. In particular, the PoNS device delivers only one kind of waveform and delivers it to all of the electrodes; this allows the PoNS device to be much smaller and simpler than the TDU, which provides for individual control of stimulation on each electrode.

To date, two major PoNS versions have been deployed, the first with a square matrix of 144 electrodes and the second with a heart-shaped matrix of 143 electrodes arranged in a hexagonal pattern. PoNS versions 1 and 2 are laboratory tools intended for investigational purposes only.

A PoNS device pending regulatory approval for commercial deployment as a medical device is under development and testing by NeuroHabilitation Corporation. Although its principle of operation is similar to that of the devices described here, its technical details are proprietary and only versions 1 and 2 of the PoNS devices are considered in the remainder of this paper.

\section{Theory of operation}

\subsection{Waveforms}

The PoNS device delivers repeating positive, capacitively-coupled, voltage-controlled pulses to the dorsal surface of the tongue via a matrix of gold-plated electrodes. Figure 2(a) shows the pulse sequence, fixed in hardware, that is delivered by each of the 16 waveform generator channels. Each channel delivers a pulse every $5 \mathrm{~ms}$ with each fourth pulse removed. This 3 -pulses-per-burst structure (i.e., the bursts repeating every $20 \mathrm{~ms}$ ) results in a comfortable, buzz-like tactile sensation that is presumed, based on earlier research [16], to have less sensory adaptation than a steady stream of pulses, although this conjecture has never been explicitly tested. The pulse sequence for each of the 16 channels begins $5 \mathrm{~ms} / 16=312.5 \mu \mathrm{s}$ after the sequence starts for the previous channel, evenly staggering the channels in time. In summary, the 16 channels in aggregate deliver 2,400 pulses every second with underlying frequencies (excluding Fourier harmonics from the rectangular pulse shape) of $50 / \mathrm{s}$ and 200/s (burst and pulse rates, respectively, per channel) and 3,200/s (representing the inter-channel delay).

\subsection{Electrodes}

For the PoNS device version 1 , the $12 \times 12$ square electrode array is subdivided into nine $4 \times 4$ blocks (Figure 3 ), each block mapping in a raster-scanned manner to the 16 waveform generator channels. Therefore, each channel connects directly to nine electrodes that 


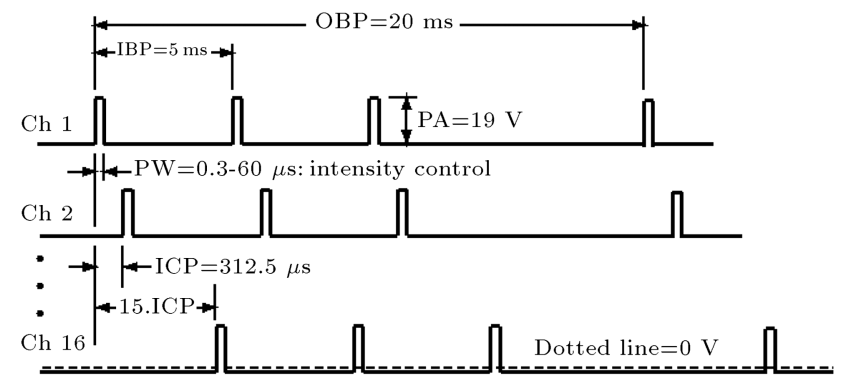

(a) Normal "Active" waveform

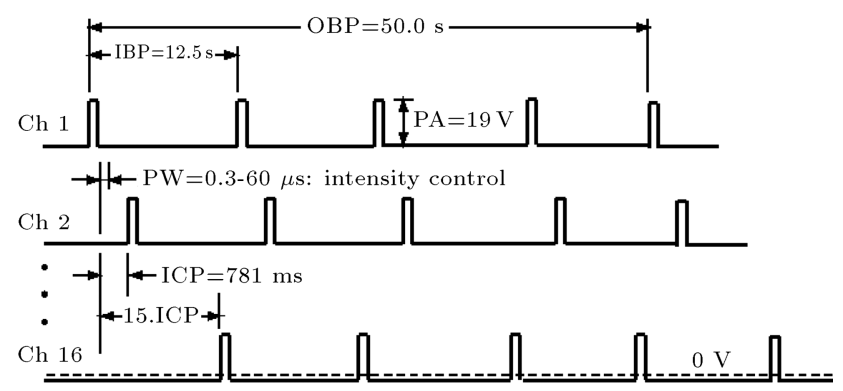

(b) "Placebo Low" waveform

Figure 2. Idealized stimulation waveforms: (a) "Normal" waveform eliciting buzz sensation, and (b) "Placebo Low" waveform which is perceivable to subjects as discrete pulses but which has much lower pulse rate (see text). Nomenclature is same as for the TDU [4]: Outer Burst Period (OBP), Inner Burst Period (IBP), Pulse Width (PW), Inner-Channel Period (ICP), and Pulse Amplitude (PA).

are simultaneously pulsed. This particular scanning order provides that each electrode stimulated should be separated from the nearest pulsed electrode by three electrodes that are unpulsed and serve as the return current path (see Section 2.3). Such separation is important to minimize potential spatial summation effects, in which current from closely-spaced electrodes may combine to activate the same afferent nerve fibers and potentially reduce effective spatial resolution.

The PoNS version 2 electrode array (Figure 4) is shaped to fit more comfortably into the mouth than the version 1 array and enable easier swallowing of saliva. Because the version 2 array is irregularly shaped, the mapping of each channel to the electrode array is likewise irregular. As discussed previously [16], the particular order of scanning is probably not critical, although an attempt is made to spatially separate simultaneously- and adjacently-pulsed electrodes as much as possible as discussed above.

Two other electrical features distinguish the version 2 array. First, because there are only 143 electrodes, channel 16 maps to only 8 rather than to 9 electrodes. Second, based on previous research showing that the electrotactile sensitivity of the tongue is not spatially uniform [17], a network of voltagedivider resistors (Figure 4) is inserted between each

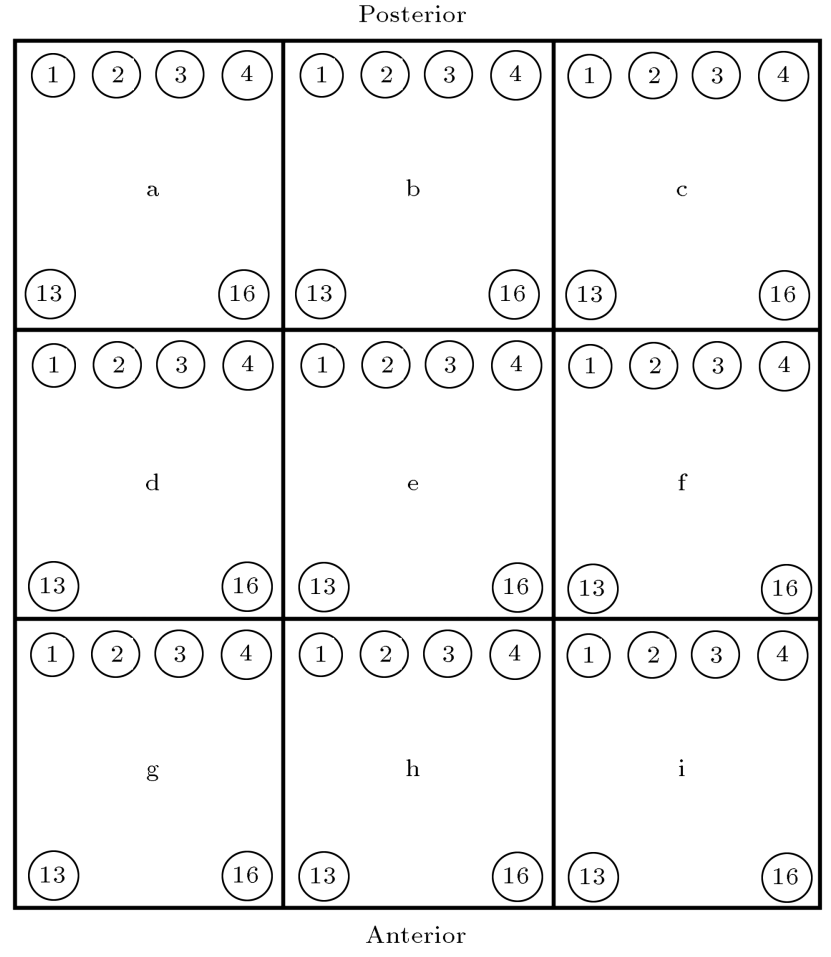

Figure 3. PoNS version 1 electrode array layout. Each of the nine blocks (a-i) comprises a square matrix of 16 electrodes (1-16). Electrodes are $1.5 \mathrm{~mm}$ in diameter with a center-center spacing of $2.3 \mathrm{~mm}$. All nine electrodes labeled " 1 " are connected to the waveform generator channel 1 and, therefore, receive a pulse simultaneously. One Inner Channel Period (ICP) later, all electrodes labeled " 2 ", receive a pulse, etc. as shown in Figure 2.

channel of the waveform generator and the corresponding electrodes. Combined with the electrode resistance (see Section 4), this voltage-divider network reduces the pulse amplitude at the more-sensitive tip and sides of the tongue, resulting in a tactile sensation that is more spatially uniform than for a matrix (such as that on PoNS version 1) receiving the same potential on all electrodes. Figure 4 illustrates the four resulting stimulation levels.

The end result of this electrode activation pattern is that the tongue receives $25,920,000$ (version 1 ) or $25,740,000$ (version 2) stimulation pulses during a typical 20-min therapy session. It is still unknown how many action potentials on afferent nerves innervating the tongue are generated by this stimulation and, furthermore, what activity they elicit in their brainstem projections. Both fingertip and lingual cutaneous mechano-afferent fibers can sustain firing rates greater than 200 pulses/s in response to mechanical stimuli $[18,19]$; data for repetitive electrical stimulation are sparse.

While the sublingual electric field distribution is likewise not well known, some reasonable bounds may be established. Surface electrode field penetration 


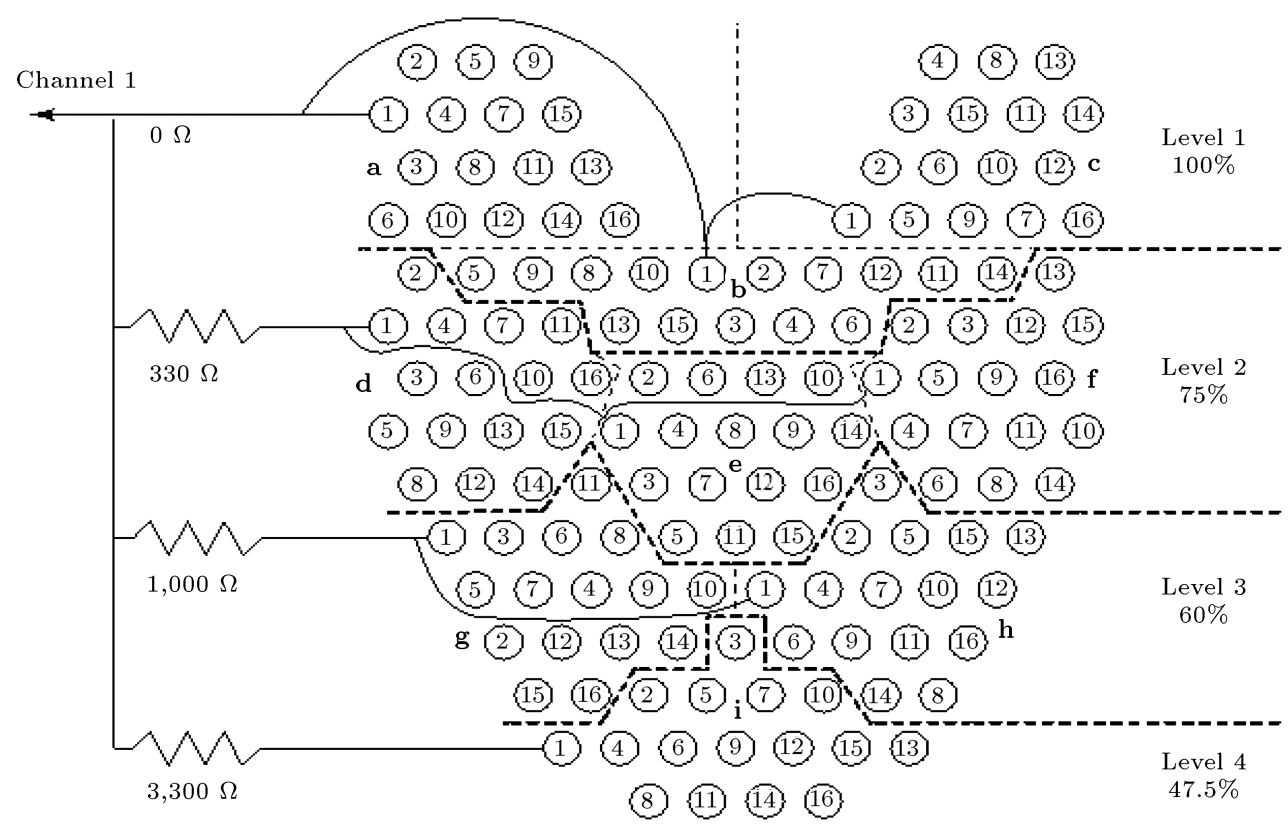

Figure 4. PoNS version 2 electrode array layout. The 1.5-mm-diameter electrodes are organized in a hexagonal layout with a center-center spacing of $2.3 \mathrm{~mm}$. The nine sectors of 16 electrodes (sector b only has 15 electrodes) pulse similarly to version 1 . The four stimulation levels, set by the series resistors, approximately compensate for spatial variations in tongue electrical sensitivity (see text). Only wiring for Channel 1 is shown; the other channels are similar. Each channel drives three electrodes with Level 1, three electrodes with Level 2, two electrodes with Level 3, and one electrode with Level 4.

is on the same order of magnitude as interelectrode spacing ([20], p. 134), in this case approximately. $1 \mathrm{~mm}$. The PoNS array field probably penetrates somewhat deeper than this owing to the relatively high resistance of the return pathway via unpulsed electrodes, relative to the highly-conductive sublingual tissue (cf. [21], Chap. 2), returning a greater proportion of current to non-adjacent electrodes and, therefore, leading to effectively greater inter-electrode spacing. Although such deeper penetration might be expected to stimulate the intrinsic tongue muscles, no obvious twitching has been observed to date.

\subsection{Output circuit}

Figure 5 illustrates the key components of the waveform generator output circuit. Although the part values are different, the essential functionality is very similar to that for the TDU and, therefore, an abbreviated summary is provided here. An analog multiplexer (Mux) distributes a fixed $19-\mathrm{V}$ potential to one of the sixteen output channels according to timing signals to its address and enable lines (not shown). These timing signals are generated by simple clock/timer/counter components.

A $1-\mu \mathrm{F}$ capacitor in series with each multiplexer output slightly shifts the mean output voltage so that the net direct current into the tongue electrode is extremely small (less than $1 \mu \mathrm{A}$, the lower limit of the author's measurement equipment), minimizing the potential for tongue irritation. Such irritation has not

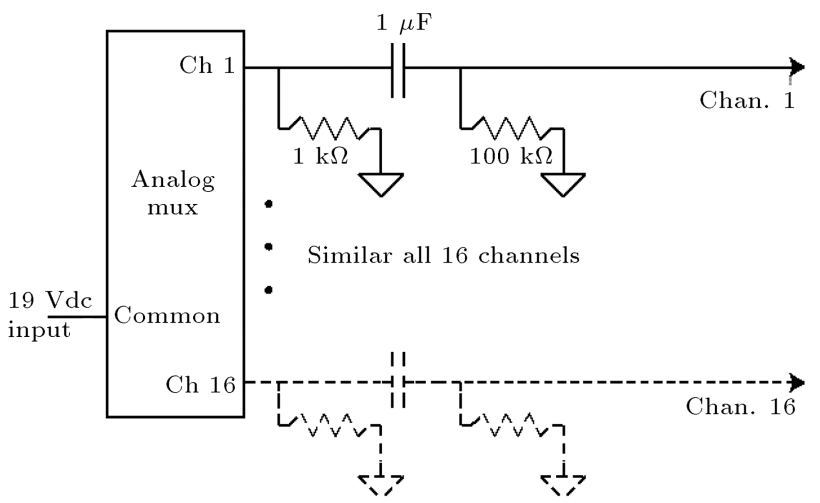

Figure 5. PoNS electrode driver circuit. An analog multiplexer (Mux) routes a fixed voltage source to one of the 16 channels according to the timing diagram in Figure 2. The capacitor provides dc blocking; the 1-k $\Omega$ resistor provides the return current path for unpulsed electrodes, and the $100-\mathrm{k} \Omega$ resistor establishes the $\mathrm{dc}$ operating point (see text). The internal "on" resistance of the multiplexer is approximately $130 \Omega$.

been reported in five years of using PoNS technology with human subjects, although a prospective long-term study has not yet been performed. This capacitance value represents a compromise between minimizing the $\mathrm{d} V / \mathrm{d} t$ voltage droop during each pulse (see Section 4) and limiting the maximal charge pulse to $19 \mu \mathrm{C}$, much lower than the $500-\mu \mathrm{C}$ upper limit recommended by Underwriters Laboratories (UL) for a $1-\mu \mathrm{F}$ capacitive discharge ([22], Figure 11.13). (The versions 1 and 2 
of PoNS devices have not been certified by UL or any other regulatory agency.)

Because the electrode array has no ground plane [23], and because inactive multiplexer outputs are open circuit, the $1-\mathrm{k} \Omega$ resistors provide the return current path (through the inactive electrodes and capacitors). The $100-\mathrm{k} \Omega$ resistors serve to stabilize the dc operating point and ensure zero mean voltage at the electrodes, but do not otherwise significantly affect the stimulation waveform.

\section{User interface}

The PoNS devices versions 1.X and 2.X have two momentary-contact push buttons for power on and power off functions. Colored lights indicate battery status (normal/low) and charge status (charging/charged). Nominal battery life is $3-4 \mathrm{~h}$. The internal lithium-polymer battery pack is charged with an external dc power supply that plugs into the $\mathrm{AC}$ mains and into the side of the PoNS unit. The PoNS device automatically turns off while being charged to prevent corded use.

Stimulation intensity control is managed differently for each PoNS version. For version 1 the user manipulates a potentiometer, which adjusts the opencircuit pulse amplitude (voltage) from 0 to $17 \mathrm{~V}$, while the pulse width is fixed at $50 \mu \mathrm{s}$. For versions 2.1 and 2.2 the user manipulates the pulse width $(0.4-60 \mu \mathrm{s})$ using a pair of UP/DOWN pushbuttons in 64 uneven increments, while the open-circuit pulse amplitude is $19 \mathrm{~V}$. Owing to the strength-duration relationship for physiological nerve stimulation, pulse amplitude or pulse width changes feel similar to the user, although minor differences in fiber recruitment patterns may be possible [24].

The PoNS device version 2.5 is programmable; pulse width automatically ramps up from a minimum to a maximum value that may be set using a simple software application on an external computer. This allows more precise control over physical stimulus levels for human subjects experiments. One limitation of such fixed levels is that the perceived level of stimulation may vary from session to session with changes in the subject's individual sensitivity to the stimulation.

\section{Electrical performance}

Figure 6 shows the typical voltage (relative to PoNS ground) and current for a representative electrode for each of the four stimulation levels for a PoNS device version 2 . The voltage trace departs from a nominallyrectangular shape because the PoNS output circuit has a finite output resistance and because the electrodeskin interface has both resistive and capacitive components [4].

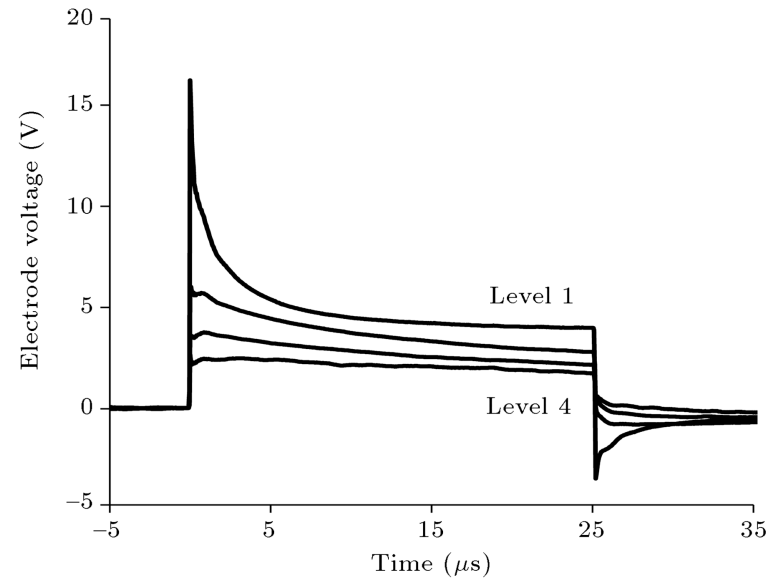

(a)

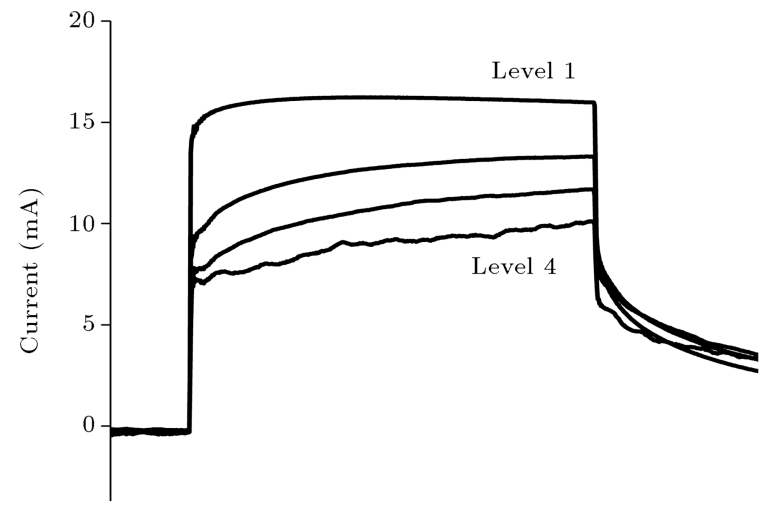

(b)

Figure 6. Representative PoNS electrode current (a) and voltage (b) waveforms. The PoNS intensity was set to deliver 25.4- $\mu$ s pulses, yielding a moderately-strong percept on the author's tongue. Preliminary waveforms recorded from other users are similar but not yet systematically explored. Current waveforms for Levels 1 , 2,3 , and 4 represent, respectively, the average values for 3 , 3, 2, and 1 electrode(s); see Figure 4. Current was measured with a Tektronix A6312 current probe (100 $\mathrm{MHz}$ ) and AM503B amplifier. All data were acquired by a $300-\mathrm{MHz}$ digital oscilloscope with a $50-\mathrm{ns}$ sample period.

Because of the capacitive component of the electrode impedance, the current exhibits an initial spike followed by an asymptotic decrease. The pulse shape difference between the four stimulation level current waveforms may be accounted for by the level-setting resistors. For the lower levels, the increasing values of the series level-setting resistors reduce both the initial current spike and the asymptotic current at the end of the pulse.

The electrode voltage waveforms are similarly affected by the level-setting resistors, but with somewhat more complex dynamics. For Level 1, the electrode voltage shows a relatively rapid asymptotic increase (the rate of which is governed by electrode capacitance), followed by a slow decrease during the pulse (due to the $\mathrm{d} V / \mathrm{d} t$ droop across the $1-\mathrm{uF}$ capacitor 
in the driver circuit). For the lower three levels, this droop is obscured by the much slower initial asymptotic increase. This is because of the larger time constant resulting from the series level-setting resistors.

Given the above comments, we would expect the afferent stimulation to be driven primarily by the charge delivered during the pulse [25]; for the four Levels $1,2,3$, and 4 , the areas under the current curve are approximately $124,93,70$, and $53 \mathrm{nC}$. These values correspond well with the target percentages shown in Figure 4 (derived from [17]), the exact distribution among the four levels being dependent on electrode impedance, which itself varies among individuals and is still under study.

Preliminary unpublished data suggest this impedance may be approximately modeled by a $1-\mathrm{k} \Omega$ resistance in series with a parallel RC network $(4.5 \mathrm{k} \Omega$, $0.5 \mathrm{nF}$ ) for the PoNS version 2 electrode array. This is slightly lower than that reported for the TDU electrode array ( $1 \mathrm{k} \Omega$ in series with $5 \mathrm{k} \Omega \| 0.5 \mathrm{nF}$ [4]), which is geometrically equivalent to the PoNS version 1 array. The greater overall electrode spatial density in the PoNS version 2 hexagonal array may account for this difference.

\section{Placebo PoNS}

Because PoNS stimulation is normally combined with therapeutic exercises [26], and because the exercises alone may result in beneficial effects, it is important to determine the relative contribution of each. While ideally we might seek a PoNS "placebo" that may be tested against the "real" device, choosing the characteristics of such a device is not obvious. For example, relative to no device at all, any purported effect might be argued to result from at least three possible causes:

1. The mechanical effect of the device in the mouth, resulting in both activation of mechanoreceptive afferents as well as user knowledge that the device is in the mouth;

2. Perception of the electrical stimulation;

3. The electrical stimulus itself, i.e. any effect of the propagated stimuli (via tongue cutaneous afferents) on brain structures that is not a result of (1) or (2).

We therefore developed two different kinds of placebo devices to enable experimental dissection of these purported effects.

\subsection{Placebo null}

The first schema, which we call Placebo Null (PN), looks like and appears to function like a normal or "Active" PoNS device but delivers no electrical stimulation. The device contains working ON and OFF buttons and indicator lights, and non-functional UP and DOWN level buttons. Removal of $+19 \mathrm{~V}$ excitation to the multiplexer (Figure 5) results in no deliberate electrical signal to the electrodes. On careful measurement, the small "noise" potentials appearing on the electrodes mostly retain the temporal characteristics of the Active device but manifest themselves as rounded pulses that are $<100 \mathrm{~ns}$ wide and $<50 \mathrm{mV}$ in amplitude, for effectively $0.17 \%$ of the minimum charge delivered by an Active device $(0.3 \mu \mathrm{s}, 19 \mathrm{~V})$, and approximately $0.029 \%$ of the sensation threshold charge for a typical user. Therefore, the user feels the device in the mouth, but does not feel or receive any effectual electrical stimulation.

\subsection{Placebo low}

To address a different concern, that subjects can feel the electrical stimulation of an Active device but do not feel it for a PN device, we developed the Placebo Low (PL). Similarly to the Active device, the PL device delivers $19-\mathrm{V}, 0.3-60-\mu$ s pulses, which at sufficient pulse width are perceivable. This stimulus, however, occurs at an extremely low rate, with each electrode receiving a pulse approximately every $12.5 \mathrm{~s}$. Because the 16 channels evenly divide this period, the user feels a stimulus somewhere on their tongue at a rate of $1.28 / \mathrm{s}$. To prevent large gaps in the sensation, the pulses occur continuously, without the burst structure used for Active devices (see Figure 2(b)). The user therefore feels a "tap-tap-tap" stimulus that moves (deterministically but seemingly randomly) around the tongue with the same sequence as for the active device; for any tap, nine electrodes are simultaneously stimulated (eight for Channel 16). Although this is different from the vibration-tingle felt for the Active device, the subject clearly knows he or she is receiving electrical stimulation, thereby allowing one to experimentally isolate the effect of feeling the stimulus. Because of the extremely low pulse rate, only 13,728 pulses are delivered to the array during a 20-min treatment session, or approximately $0.053 \%$ relative to Active. Although the dose-response characteristic for PoNS stimulation is unknown and possibly nonlinear, it is reasonable to assume based on this large ratio that any neuromodulatory effect resulting from cutaneous afferent stimulation is much smaller for PL than for Active devices.

\section{Application to neurorehabilitation}

The PoNS device has enabled a number of studies examining the use of tongue stimulation to enhance neurorehabilitation. Those with at least partial results published or presented are briefly summarized next, divided into three categories: etiologies, symptoms, and placebo control.

Previous sensory substitution research showed 
that tongue-based vestibular feedback improved a number of neurological symptoms, including particularly balance and gait, for individuals with a variety of peripheral and central vestibular disorders [5,6,27-33]. This etiology independence suggests that purported beneficial effects of information-free tongue neurostimulation, such as that delivered by the PoNS device, may similarly be etiology independent. Research in progress has provided initial evidence that this may indeed be the case; efficacy has been observed in preliminary studies investigating multiple sclerosis [34,35], traumatic brain injury [36-41], stroke [41-46], Parkinson's disease $[47,48]$ spinal cord injury [49], and posterior fossa syndrome [50].

The preliminary efficacy of PoNS stimulation and related therapeutic exercises have also been observed across a variety of symptoms including not only balance and gait (above) but also control of eye movements $[38,42,44,48,51,52]$, muscle activation patterns [36-38], cognitive function [34,37], self-reports of sleep, bowel and bladder function, concentration, and tremor [53]. These observations, coupled with results across etiologies above, have led to formulation of a conceptual framework for generalized neurorehabilitation called cranial-nerve noninvasive neuromodulation, or CN-NINM [26,54-57].

A limitation of most of the studies cited above is lack of a control group. One controlled study, however, showed that gait in a group of individuals with moderate (cane/walker) stage multiple sclerosis improved more for a group of 10 subjects using an active PoNS device than for a control group of 10 subjects using a Placebo Null PoNS device [35]. This benefit extended into the 12-week at-home phase of the study following the 2 -week in-lab phase, showing the potential for home-based PoNS therapy. Because both groups received the benefit of balance and gait exercises as well as any purported psychological benefit of having a PoNS device in their mouth, it would appear that the electrical stimulus in the active group resulted in the difference. Because, however, the active group felt the stimulus and the control group did not, it is not yet possible (on the basis of the intergroup difference) to differentiate between a potential psychological benefit of feeling the stimulation and a potential neurological benefit of subjects receiving the stimulation. The Placebo Low PoNS device will help to resolve this question, and is included in a study currently in progress at the University of WisconsinMadison investigating rehabilitation of balance and gait in mild-to-moderate traumatic brain injury.

\section{Disclosure}

The technology and application of the PoNS device are covered by US Patents 8,849,407, 8,909,345, 9,020,612,
$9,597,501,9,597,504$, and 9,656,078, and by other patents pending. The author has an ownership interest in Advanced NeuroRehabilitation LLC, a company formed to commercialize non-invasive neuromodulation technology, and which receives royalty payments for the aforementioned patents. The author also has an ownership interest in Helius Medical Technologies, a company formed to commercialize rehabilitation technologies, and which controls the PoNS trademark.

\section{Acknowledgments}

This work was supported by gifts to the University of Wisconsin Foundation. We are particularly grateful for gifts from Norm Flodine and an anonymous donor.

The author acknowledges the technical design assistance of John Peterman at Simplex Scientific, and the collaboration of research colleagues Yuri Danilov and Mitchell Tyler in developing therapeutic applications for the PoNS device.

\section{Acronyms and abbreviations}

CN-NINM Cranial-Nerve NonInvasive

NeuroModulation

IBP Inner Burst Period

ICP Inner Channel Period

Mux Analog Multiplexer

OBP Outer Burst Period

PA Pulse Amplitude

PoNS Portable Neuromodulation Stimulator

PL Placebo Low

PN Placebo Null

PW Pulse Width

TDU Tongue Display Unit

UL Underwriters Laboratories

\section{References}

1. Bach-y-Rita, P. and Tyler, M.E. "Tongue manmachine interface", In Medicine Meets Virtual Reality Conf., Amsterdam, pp. 17-19 (2000).

2. Bach-y-Rita, P. and Kaczmarek, K.A. "Tongue placed tactile output device", USA Patent 6,430,450 (2002).

3. Bach-y-Rita, P., Tyler, M.E. and Kaczmarek, K.A. "Seeing with the brain", Int. J. Hum. Comp. Interact., 15(2), pp. 285-295 (2003).

4. Kaczmarek, K.A. "The tongue display unit for electrotactile spatiotemporal pattern presentation", Scientia Iranica D, 18(6), pp. 1476-1485 (2011).

5. Tyler, M., Danilov, Y. and Bach-y-Rita, P. "Closing an open-loop control system: Vestibular substitution through the tongue", J. Integrat. Neurosci., 2(2), pp. 159-164 (2003). 
6. Danilov, Y.P., Tyler, M.E., Skinner, K.L., Hogle, R.A. and Bach-y-Rita, P. "Efficacy of electrotactile vestibular substitution in patients with peripheral and central vestibular loss", J. Vestib. Res., 17(2-3), pp. 119-130 (2007).

7. Danilov, Y.P., Tyler, M.E., Wildenberg, J.C. and Meyerand, M.E. "Neural pathways in cranial nerve non-invasive neuromodulation (CN-NINM) (abstract of poster)", In Soc. Neurosci. Annu. Mtg. (2008).

8. Tyler, M.E., Danilov, Y.P., Wildenberg, J.C. and Meyerand, M.E. "Neural pathways in non-invasive neuromodulation (abstract of poster)", In Society for Neurosci. Annu. Mtg. (2008).

9. Wildenberg, J.C., Tyler, M.E., Danilov, Y.P. and Meyerand, M.E. "Cranial-nerve non-invasive neuromodulation (CN-NINM) effects on cortical and subcortical activity as measured with BOLD-fMRI (abstract of poster)", In Soc. Neurosci. 2008 Annu. Mtg. (2008).

10. Wildenberg, J., Meier, T., Nair, V., Tyler, M., Danilov, Y., Kaczmarek, K.A. and Meyerand, M. "Altered connectivity after electrical tongue stimulation as measured by dynamic causal modeling (poster)", In 16th Annual Meeting of the Organization for Human Brain Mapping, Barcelona, Spain (2010).

11. Wildenberg, J.C., Tyler, M.E., Danilov, Y.P., Kaczmarek, K.A. and Meyerand, M.E. "Sustained cortical and subcortical neuromodulation induced by electrical tongue stimulation", Brain Imag. Behav., 4, pp. 199211 (2010).

12. Wildenberg, J., Tyler, M., Danilov, Y., Kaczmarek, K., Kuo, J. and Meyerand, M.E. "High-resolution fMRI detects activity and neuromodulation of individual brainstem nuclei", In 2011 AANS Annu Meeting, Denver, pp. A438-A439 (2011).

13. Wildenberg, J.C., Tyler, M.E., Danilov, Y.P., Kaczmarek, K.A. and Meyerand, M.E. "High-resolution fMRI detects neuromodulation of individual brainstem nuclei by electrical tongue stimulation in balanceimpaired individuals", Neuroimage, 56, pp. 2129-2137 (2011).

14. Wildenberg, J.C., Tyler, M.E., Danilov, Y.P., Kaczmarek, K.A. and Meyerand, M.E. "Electrical tongue stimulation normalizes activity within the motionsensitive brain network in balance-impaired subjects as revealed by group independent component analysis", Brain Connectivity, 1(3), pp. 255-265 (2011).

15. Wildenberg, J.C., Tyler, M.E., Danilov, Y.P., Kaczmarek, K.A. and Meyerand, M.E. "Altered connectivity of the balance processing network after electrical tongue stimulation in balance-impaired individuals", Brain Connectivity, 3(1), pp. 87-97 (2013).

16. Kaczmarek, K.A. "Electrotactile adaptation on the abdomen: Preliminary results", IEEE Trans. Rehab. Eng., 8(4), pp. 499-505 (2000).

17. Tyler, M.E., Braun, J.G. and Danilov, Y.P. "Spatial mapping of electrotactile sensation threshold and intensity range on the human tongue: Initial results", In
Proc. IEEE Eng. Med. Biol. Soc., Minneapolis, MN, pp. 559-562 (2009).

18. Talbot, W.H., Darian-Smith, I., Kornhuber, H.H. and Mountcastle, V.B. "The sense of flutter-vibration: Comparison of the human capacity with response patterns of mechanoreceptive afferents from the monkey hand", J. Neurophys., 31, pp. 301-334 (1968).

19. Trulsson, M. and Essick, G.K. "Low-threshold mechanoreceptive afferents in the human lingual nerve", J. Neurophys., 77(2), pp. 737-748 (1997).

20. Grimnes, S. and Martinsen, O.G., Bioimpedance and Bioelectricity Basics, New York, Academic Press (2000).

21. Prior, R.E., Study of Electrocutaneous Parameters Relevant to Dynamic Tactual Communication Systems, University of California, Los Angeles (1972).

22. Reilly, J.P., Applied Bioelectricity, New York, Springer (1998).

23. Kaczmarek, K.A. and Tyler, M.E. "Effect of electrode geometry and intensity control method on comfort of electrotactile stimulation on the tongue", In Proc. ASME Dyn. Sys. Contr. Div., Orlando, Florida, pp. 1239-1243 (2000).

24. Grill, W.M. and Mortimer, J.T. "Stimulus waveforms for selective neural stimulation", IEEE Eng. Med. Biol. Mag., 14(4), pp. 375-385 (July, 1995).

25. Mortimer, J.T. "Electrical excitation of nerve", Neural Porstheses: Fundamental Studies, Biophysics and Bioengineering Series, W.F. Agnew and D.B. McCreery, Eds., pp. 67-83, Englewood Cliffs, NJ: Prentice Hall (1990).

26. Danilov, Y., Kaczmarek, K., Skinner, K. and Tyler, M. "Cranial nerve noninvasive neuromodulation: New approach to neurorehabilitation", Brain Neurotrauma: Molecular, Neuropsychological and Rehabilitation Aspects, F.H. Kobeissy and S.W. Hoffman, Eds., pp. 605627: CRC Press (2015).

27. Barros, C.G., Bittar, R.S. and Danilov, Y. "Effects of electrotactile vestibular substitution on rehabilitation of patients with bilateral vestibular loss", Neurosci. Letters, 476(3), pp. 123-126 (2010).

28. Danilov, Y.P., Tyler, M.E. and Kaczmarek, K.A. "Vestibular sensory substitution using tongue electrotactile display", Human Haptic Perception: Basics and Applications, M. Grunwald, Ed., pp. 467-480, Basel, Switzerland, Birkhauser Verlag (2008).

29. Polat, S. and Uneri, A. "Vestibular substitution: comparative study", J. Laryngol. Otol., 124, pp. 852-858 (2010).

30. Wood, S.J., Black, F.O., MacDougall, H.G. and Moore, S.T. "Electrotactile feedback of sway position improves postural performance during galvanic vestibular stimulation", Basic And Clinical Aspects of Vertigo and Dizziness, 1164, pp. 492-498 (2009).

31. Uneri, A. and Polat, S. "Vestibular rehabilitation with electrotactile vestibular substitution: early effects", 
Eur. Arch. Otorhinolaryngol., 266, pp. 1199-1203 (2009).

32. Vuillerme, N., Nicolas, P., Chenu, O., Demongeot, J., Payan, Y. and Danilov, Y. "Sensory supplementation system based on electrotactile tongue biofeedback of head position for balance control", Neurosci. Letters, 431(3), pp. 206-210 (2008).

33. Vuillerme, N. and Cuisinier, R. "Head position-based electrotactile tongue biofeedback affects postural responses to Achilles tendon vibration in humans", Exp. Brain Res., 186(3), pp. 503-508 (Apr. 2008).

34. Danilov, Y., Tyler, M., Kaczmarek, K., Rust, K. and Subbotin, A. "Neurorehabilitation of Multiple Sclerosis symptoms using cranial nerve non-invasive neuromodulation (CN-NINM): controlled study", In Proc. Soc. Neurosci. Annu. Mtg., Washington, DC, pp. 253.10 (poster) (2011).

35. Tyler, M.E., Kaczmarek, K.A., Rust, K.L., Subbotin, A.M., Skinner, K.L. and Danilov, Y.P. "Non-invasive neuromodulation to improve gait in chronic multiple sclerosis: a randomized double blind controlled pilot trial", J. NeuroEng. Rehab., 11(79), pp. 1-10 (2014).

36. Danilov, Y.P., Tyler, M.E. and Kaczmarek, K.A. "New approach to chronic TBI rehabilitation: cranial nerve noninvasive neuromodulation (CN-NINM technology)", In Neurotrauma 2012, Phoenix, AZ (2012).

37. Danilov, Y., Subbotin, A., Skinner, K., Verbny, Y., Kaczmarek, K. and Tyler, M. "Cranial nerve noninvasive neuromodulation for symptomatic treatment of mild and moderate traumatic brain injury", In Proc. 31st Annu. Nat. Neurotrauma Symp., Nashville, TN (2013).

38. Danilov, Y., Subbotin, A., Skinner, K., Verbny, Y., Kaczmarek, K. and Tyler, M. "Cranial nerve noninvasive neuromodulation for symptomatic treatment of mild and moderate traumatic brain injury (poster)", In Proc. 10th World Congress Brain Injury, San Francisco (2014).

39. Liegl, K.P., Rust, K.L. and Smith, R.O. "Introduction to the portable neuromodulation stimulator (PoNS$\mathrm{tm})$ device and effects on balance and gait for individuals with traumatic brain injuries", In Proc. Rehab. Eng. Assist. Tech. Soc. North Amer. Annu. Conf., Seattle, WA (2013).

40. Danilov, Y., Subbotin, A., Skinner, K., Verbny, Y., Kaczmarek, K. and Tyler, M. "Cranial nerve non-invasive neuromodulation for symptomatic treatment of mild and moderate traumatic brain injury (poster)", In Proc. 31st Annu. Nat. Neurotrauma Symp., Nashville, TN (2013).

41. Kukurin, G.W. "Non-invasive brain stimulation in mTBI: A case series (Poster)", In 10th World Congress on Brain Injury (2014).

42. Verbny, Y., Skinner, K., Kaczmarek, K. and Danilov, Y. "Effects of CN-NINM Intervention on eye movement rehabilitation in chronic stroke: a case study", In Proc. Soc. Neurosci. Annu. Mtg., San Diego (2013).
43. Kukurin, G.W. "A case of traumatic brain injury (TBI) with persistent post-concussion symptoms: Response to cranial nerve non-invasive neuromodulation (CN-NINM)", In Abstracts of the 6th Pannonian Symp. CNS Injury, Pecs, Hungary, p. 133 (2013).

44. Verbny, Y., Skinner, K., Tyler, M., Kaczmarek, K. and Danilov, Y. "Eye movement rehabilitation by CN-NINM intervention in chronic stroke", In ARVO Conference, Orlando, FL (2014).

45. Danilov, Y., Skinner, K., Tyler, M. and Verbny, Y. "Effects of CN-NINM intervention on eye movement rehabilitation in chronic Stroke: A pilot study", In Mechanisms of Action Conference, Orlando, FL (2014).

46. Danilov, Y., Skinner, K., Subbotin, A., Verbny, Y., Tyler, M. and Kaczmarek, K. "Effects of CN-NINM intervention on chronic stroke rehabilitation: A case study", In Society for Neuroscience Annual Meeting, San Diego (2013).

47. Danilov, Y., Tyler, M., Kaczmarek, K.A. and Luzzio, C. "Rehabilitation of multiple sclerosis and Parkinson's symptoms using cranial nerve non-invasive neuromodulation (CN-NINM)", In Soc. Neurosci. Annu. Mtg. (2010).

48. Verbny, Y., Vishwanathan, G., Skinner, K., Tyler, M., Kaczmarek, K. and Danilov, Y. "Eye movement enhancement in Parkinson's disease as a result of CNNINM intervention: a case study", In $A R V O$, Denver, CO (2015).

49. Chisholm, A.E., Malik, R.N., Blouin, J.S., Borisoff, J., Forwell, S. and Lam, T. "Feasibility of sensory tongue stimulation combined with task-specific therapy in people with spinal cord injury: a case study", $J$. NeuroEng. Rehab, 11(96), pp. 1-9 (2014).

50. Harbourne, R., Becker, K., Arpin, D.J., Wilson, T.W. and Kurz, M.J. "Improving the motor skill of children with posterior fossa syndrome: a case series", Pedriac Phys. Ther., 26(4), pp. 462-468 (2014).

51. Danilov, Y.P., Verbny, Y.I., Skinner, K.L., Tyler, M.E. and Kaczmarek, K.A. "Eye movement rehabilitation using tongue stimulation (CN-NINM technology): A set of case studies", In 12th World Congr. Int. Neuromod. Soc., Montreal, pp. ID\#2224797 (2015).

52. Danilov, Y.P., Verbny, Y.I., Skinner, K.L., Tyler, M.E. and Kaczmarek, K.A. "Eye movement rehabilitation by CN-NINM intervention: a set of case studies", In NABIS Conference, San Antonio (2015).

53. Danilov, Y., Tyler, M., Kaczmarek, K.A. and Luzzio, C. "Rehabilitation of multiple sclerosis symptoms using cranial nerve non-invasive neuromodulation (CNNINM) (abstract of poster)", In Soc. Neurosci. Annu. Mtg. (2009).

54. Danilov, Y.P., Tyler, M.E. and Kaczmarek, K.A. "Cranial-nerve non-invasive neuromodulation (CNNINM): New approach to neurorehabilitation (abstract of poster)", In Int. J. Psychophysiol., pp. 301302 (2008). 
55. Danilov, Y.P., Tyler, M.E., Kaczmarek, K.A. and Skinner, K.L. "New approach to neurorehabilitation: Cranial nerve noninvasive neuromodulation (CNNINM) technology", In Proc. SPIE (2014).

56. Danilov, Y.P. and Kublanov, V.S. "Emerging noninvasive neurostimulation technologies: CN-NINM and SYMPATOCORECTION", J. Behav. Brain. Sci., 4, pp. 105-113 (March, 2014).

57. Papa, L., LaMee, A., Tan, C.N. and Hill-Pryor, C. "Systematic review and meta-analysis of noninvasive cranial nerve neuromodulation for nervous system disorders", Arch. Phys. Med. Rehabil., 95(12), pp. 2435-2543 (2014).

\section{Biography}

Kurt Alan Kaczmarek received a BS degree from the University of Illinois, Urbana, in 1982, and MS and $\mathrm{PhD}$ degrees from the University of WisconsinMadison, Madison, in 1984 and 1991, respectively, all in Electrical Engineering. From 1984 to 1986, he was a Senior Engineer with Baxter International, Deerfield, IL. He is currently a Senior Scientist and a laboratory Co-director at the University of Wisconsin, Madison, where, since 1992, he has been studying the mechanisms and perception of electrical stimulation of touch. His current research interests include tactile displays, sensory rehabilitation and augmentation, and neurorehabilitation. 